Geom. Funct. Anal. Vol. 20 (2010) 571-591

DOI 10.1007/s00039-010-0068-5

Published online June 10, 2010

(C) 2010 The Author(s).

GAFA Geometric And Functional Analysis

This article is published with open access at Springerlink.com

\title{
MANIFOLDS WITH 1/4-PINCHED FLAG CURVATURE
}

\author{
LEI Ni AND BURKHARD WILKING
}

\begin{abstract}
We say that a nonnegatively curved manifold $(M, g)$ has quarter-pinched flag curvature if for any two planes which intersect in a line the ratio of their sectional curvature is bounded above by 4 . We show that these manifolds have nonnegative complex sectional curvature. By combining with a theorem of Brendle and Schoen it follows that any positively curved manifold with strictly quarter-pinched flag curvature must be a space form. This in turn generalizes a result of Andrews and Nguyen in dimension 4. For odd-dimensional manifolds we obtain results for the case that the flag curvature is pinched with some constant below one quarter, one of which generalizes a recent work of Petersen and Tao.
\end{abstract}

\section{Introduction}

Let $(M, g)$ be a Riemannian manifold with curvature tensor $R$ and curvature operator $\operatorname{Rm}$ (we follow the convention $R(X, Y, Z, W)=\langle\operatorname{Rm}(X \wedge Y), Z \wedge W\rangle)$. Assume that $(M, g)$ has nonnegative sectional curvature. Fixing a point $x \in M$, for any nonzero vector $e \in T_{x} M$, we define the flag curvature in the direction $e$ by the symmetric bilinear form $R_{e}(X, X)=R(e, X, e, X)$. Restrict $R_{e}(\cdot, \cdot)$ to the subspace orthogonal to $e$, it is semi-positive definite. We call $(M, g)$ has $\lambda$-pinched flag curvature $(1>\lambda \geq 0)$ if the eigenvalues of the symmetric bilinear form $R_{e}(\cdot, \cdot)$, restricted to the subspace orthogonal to $e$, are $\lambda$-pinched for all nonzero vector $e$. Namely

$$
R_{e}(X, X) \geq \lambda(x) R_{e}(Y, Y)
$$

for any $X, Y$ in the subspace orthogonal to $e$, with $|X|=|Y|$. This condition was recently brought to attention by the work of Andrews and Nguyen [AnN]. In [AnN], Andrews and Nguyen proved the following theorem.

Theorem (Andrews-Nguyen). For any $\lambda \geq 1 / 4$ the class of positively curved 4manifolds with $\lambda$-pinched flag curvature is invariant under the Ricci flow. Moreover any such manifold is either diffeomorphic to a spherical space form or isometric to $\mathbb{C} P^{2}$ with Fubini-Study metric (up to a scaling).

The $\lambda$-pinched flag curvature condition is equivalent to saying that $K\left(\sigma_{1}\right) \geq$ $\lambda K\left(\sigma_{2}\right)$ for a pair of planes $\sigma_{1}$ and $\sigma_{2}$ such that $\sigma_{1} \cap \sigma_{2} \neq\{0\}$. The above result generalizes a theorem of Chen [C], who used Hamilton's Ricci flow to classify four

Keywords and phrases: Ricci flow, curvature pinching, complex sectional curvature 2010 Mathematics Subject Classification: Primary 53C20; Secondary 53C44 
manifolds with pointwise quarter-pinched sectional curvature. We recall that a manifold is said to be pointwise quarter pinched if $K\left(\sigma_{1}\right) \geq \frac{1}{4} K\left(\sigma_{2}\right)$ holds for all planes $\sigma_{1}, \sigma_{2} \subset T_{x} M$ and all $x \in M$.

The earlier convergence results on Ricci flow are mostly for manifolds with dimension not greater than four $[\mathrm{H} 1,2]$. Recently, in [BöW2], Böhm and the second author constructed a new family of cones (in the space of algebraic curvature operators) which is invariant under Ricci flow and proved that any compact manifold with 2-positive curvature operator is diffeomorphic to a spherical space form, by showing that the normalized Ricci flow evolves such a metric to one with constant curvature.

Soon afterwards, Brendle and Schoen [BrS1] proved that any manifold with strictly quarter-pinched sectional curvature is a spherical space form. The key novel step in the proof is to show that nonnegative isotropic curvature is invariant under the Ricci flow, which was also independently proved by Nguyen [N]. Another important step was to show that for any pointwise $1 / 4$-pinched manifold $M$, the manifold $(M, g) \times \mathbb{R}^{2}$ has nonnegative isotropic curvature. The convergence of the normalized Ricci flow for metrics with strictly 1/4-pinched sectional curvature then follows from a convergence result in [BöW2] (cf. Theorem 3.2 in section 3). Later on it was pointed out in $[\mathrm{NiW}]$ that $(M, g) \times \mathbb{R}^{2}$ has nonnegative isotropic curvature if and only if $(M, g)$ has nonnegative complex sectional curvature. (The readers should consult [BöW2], [BrS1], and section 2 for the notation involved.) The convergence result of $[\mathrm{BrS} 1]$ can then be restated as

Theorem (Brendle-Schoen). A metric $g$ of positive complex sectional curvature on a compact manifold evolves under the normalized Ricci flow to a constant curvature limit metric.

The part that strictly pointwise 1/4-pinched manifolds have positive complex sectional curvature was essentially known to be true by an argument of [YZ] and [He] for the negatively 1/4-pinched manifolds. The main result of this paper is to generalize the latter result by relaxing the assumption of pointwise $1 / 4$-pinched sectional curvature to the assumption of 1/4-pinched flag curvature.

Theorem 1.1. Let $\left(M^{n}, g\right)$ be a nonnegatively curved Riemannian manifold. If $(M, g)$ has quarter-pinched flag curvature, then $(M, g)$ has nonnegative complex sectional curvature.

This is proved in section 2. Combining the last two results and the rigidity theorem of $[\mathrm{BrS} 2]$ on manifolds with weakly 1/4-pinched sectional curvature, we obtain the following corollary as a generalization.

COROLlary 1.2. Let $\left(M^{n}, g\right)$ be a compact nonnegatively curved Riemannian manifold with 1/4-pinched flag curvature and the scalar curvature $\operatorname{Scal}(x)>0$ for some $x \in M$. Then $(M, g)$ is diffeomorphic to a spherical space form or isometric to finite quotient of a rank-one symmetric space.

This is done in section 3 . We should point out that if an algebraic curvature operator has $\lambda$-pinched flag curvature, then its sectional curvature is $\lambda^{2}$-pinched. We will see in section 4 that this inequality is indeed sharp in dimensions above 3 . 
A result similar to Theorem 1.1 yields the following generalization of a result by Yau and Zheng [YZ], and Hernández [He], by relaxing the condition on 1/4-pinched sectional curvature to 1/4-pinched flag curvature.

Theorem 1.3. If $\left(M^{m}, g\right)(m \geq 2)$ is a compact complex Kähler manifold which also admits a Riemannian metric $h$ with negative 1/4-pinched flag curvature. Then $(M, h)$ must be holomorphically isometric to a compact quotient of the unit ball $\mathbb{B}^{m} \subset \mathbb{C}^{m}$.

This is also done in section 4 . In section 5 we consider nonnegatively curved manifolds of odd dimension $n=2 m+1$ with $\lambda$-pinched flag curvature for $\lambda<1 / 4$. A generalization of an earlier result of Berger [B1] on the vanishing of the second Betti number is obtained for $\lambda \geq \frac{n-3}{4 n-9}$ (cf. Theorem 5.1), provided that the scalar curvature $\operatorname{Scal}(x)>0$ for some $x \in M$. An interesting corollary is for 5 -dimensional manifolds.

Corollary 1.4. If $(M, g)$ is a closed positively curved 5-manifold with 2/11pinched flag curvature, then $M$ must be a rational-homological sphere.

An open question is whether or not a 5-manifold as above is diffeomorphic to a spherical space form. For odd-dimensional manifolds, we also proved a result generalizing an earlier one of Petersen and Tao [PT] for nonnegatively curved manifolds (even orbifolds) with flag pinching constant below one quarter and an arbitrarily small global curvature pinching condition.

Theorem 1.5. For any dimension $n \geq 4$ and $C>0$, there is an $\varepsilon>0$ such that the following holds. Let $\left(M^{n}, g\right)$ be a nonnegatively curved Riemannian orbifold of dimension $n$ with $\frac{1-\varepsilon}{4}$ pinched-flag curvature and scalar curvature satisfying $1 \leq$ Scal $\leq C$. Then the following holds:

(i) When $n=2 m+1, M$ admits a metric of constant curvature;

(ii) When $n=2 m$, either $M$ is diffeomorphic to the quotient of a rank-one symmetric space by a finite isometric group action or it is diffeomorphic to the quotient of a weighted complex projective space by a finite group action.

This was done in section 6 . The proof, in the odd dimensions, makes use of the above mentioned generalization (of an earlier result of Berger) on the vanishing of the second Betti number as well as the injectivity radius estimates of PetruninTuschmann and Fang-Rong $[\mathrm{PeT}],[\mathrm{FR}])$. We also explain that in even dimensions for $\varepsilon>0$ there are infinitely many distinct simply connected orbifolds (namely certain weighted projective space) with curvature $\frac{1-\varepsilon}{4}<K<1$. Nevertheless we are able to classify orbifolds with nearly quarter-flag pinching in even dimensions as well. This might be interesting in other context as well since its proof provides, in very special circumstances, a method to deal with a sequence of collapsing solutions of the Ricci flow.

\section{Pinched Flag Curvature and the Complex Sectional Curvature}

Fix a point $x \in M$. Let $T_{x}^{\mathbb{C}} M \doteqdot T_{x} M \otimes \mathbb{C}$ be the complexified tangent bundle. The curvature operator, viewed as a symmetric tensor (transformation) of $\wedge^{2} T_{x} M$, can be 
extended to $\wedge^{2} T_{x}^{\mathbb{C}} M$. We also extend the inner product $\langle\cdot, \cdot\rangle$ bilinearly to $T_{x}^{\mathbb{C}} M$. We defined the complex sectional curvature of a pair of vectors $U, V$ by $R(U, V, \bar{U}, \bar{V}) \doteqdot$ $\langle\operatorname{Rm}(U \wedge V), \overline{U \wedge V}\rangle$. This notion of curvature appeared previously in geometry mainly in the study of harmonic maps and its applications to the rigidity theorems (cf. [S], [Si]). The interested reader may also consult $[\mathrm{G}]$ for its relations with various other curvature notions. In particular, we say that $(M, g)$ has nonnegative isotropic curvature if $R(U, V, \bar{U}, \bar{V}) \geq 0$ for any $U$ and $V$ which spans an isotropic (with respect to $\langle\cdot, \cdot\rangle)$ plane. If $U=X+\sqrt{-1} Y, V=Z+\sqrt{-1} W$, via the first Bianchi identity, we have that

$$
\begin{array}{r}
R(U, V, \bar{U}, \bar{V})=R(X, Z, X, Z)+R(Y, W, Y, W)+R(X, W, X, W)+R(Y, Z, Y, Z) \\
-2 R(X, Y, Z, W) .
\end{array}
$$

The aim of this section is to show that $\lambda$-pinched flag curvature implies the positivity of the complex sectional curvature for $\lambda>1 / 4$. The proof relies on an improved version of Berger's lemma [B1] and a modification of the argument of [YZ]. (See also $[\mathrm{He}],[\mathrm{NiW}]$.

First we start with a simple known lemma. We include its proof for the sake of the completeness.

LEMMA 2.1.

$$
\begin{aligned}
6 R(X, Y) Z=-R(Y, Z+ & X)(Z+X)+R(Y, Z-X)(Z-X) \\
& +R(X, Z+Y)(Z+Y)-R(X, Z-Y)(Z-Y) .
\end{aligned}
$$

Proof. First Bianchi yields

$$
-R(X, Y) Z=R(Z, X) Y+R(Y, Z) X .
$$

While

$$
\begin{aligned}
R(Y, Z) X & =\frac{1}{2}(R(Y, Z+X)(Z+X)-R(Y, Z-X)(Z-X))-R(Y, X) Z \\
R(Z, X) Y & =-R(X, Z) Y \\
& =-\frac{1}{2}(R(X, Z+Y)(Z+Y)-R(X, Z-Y)(Z-Y))+R(X, Y) Z
\end{aligned}
$$

Combining them we have the claimed equation.

Corollary 2.1. Let $k(A, B) \doteqdot R(A, B, A, B)$.

$$
-R(X, Y, Z, W)=\frac{1}{12}\left(\begin{array}{l}
-k(Y+W, Z+X)+k(Y, Z+X)+k(W, Z+X) \\
-k(X+W, Z-Y)+k(X, Z-Y)+k(W, Z-Y) \\
-k(Y-W, Z-X)+k(Y, Z-X)+k(W, Z-X) \\
-k(X-W, Z+Y)+k(X, Z+Y)+k(W, Z+Y)
\end{array}\right) .
$$

Proof. By the lemma we have that

$$
\begin{array}{r}
-R(X, Y, Z, W)=\frac{1}{6}(R(Y, Z+X, Z+X, W)-R(Y, Z-X, Z-X, W) \\
-R(X, Z+Y, Z+Y, W)+R(X, Z-Y, Z-Y, W)) .
\end{array}
$$

The result follows from the fact that $R(\cdot, Z+X, Z+X, \cdot), R(\cdot, Z-X, Z-X, \cdot)$, etc., are symmetric bilinear forms and the standard polarization formula.

The next lemma is the key to estimate the complex sectional curvature in terms of the pinching constant of the flag curvatures. 
Lemma 2.2. Let $e \in T_{x} M$ be a nonzero vector. Assume that the sectional curvature is nonnegative at $x$. Let $b(\cdot, \cdot) \doteqdot R_{e}(\cdot, \cdot)$, and let $\mathcal{N}$ be the subspace orthogonal to $e$. Assume that the flag curvature of $e$ is $\lambda$-pinched with $\lambda>0$. Then for any $Y$, $W \in \mathcal{N}$ such that $\langle Y, W\rangle=0$,

$$
\frac{2 \lambda}{1+\lambda}(b(Y, Y)+b(W, W)) \leq b(Y+W, Y+W) \leq \frac{2}{1+\lambda}(b(Y, Y)+b(W, W)) .
$$

If equality holds and $b \neq 0$, then $|W|=|Y|$.

Proof. We may restrict $b(\cdot, \cdot)$ to the 2-plane $\mathcal{P}_{2}$ spanned by $Y, W$. We also may assume $\lambda$ is the largest number such that $b$ restricted to this two-dimensional subspace is $\lambda$-pinched. In fact, otherwise we increase $\lambda$ and prove inequality (2.3) with the improved $\lambda$. Assume that $b\left(e_{1}, e_{1}\right)=\max _{Z \in \mathcal{P}_{2},|Z|=1} b(Z, Z)=\hat{\Lambda}$ and $b\left(e_{2}, e_{2}\right)=\min _{Z \in \mathcal{P}_{2},|Z|=1} b(Z, Z)=\hat{\lambda}$. Without loss of the generality we can assume that $\hat{\lambda}>0$. Let $a:=1 / \lambda=\hat{\Lambda} / \hat{\lambda}$. Since one can multiply both sides of (2.3) by a constant, without loss of the generality we can assume that $|Y|=1$ and $|W| \leq 1$. Write $Y=\cos \theta e_{1}+\sin \theta e_{2}$ and $W=\eta\left(-\sin \theta e_{1}+\cos \theta e_{2}\right)$ with $|\eta|<1$. For the upper bound we just compute $b(Y+W, Y+W)$ and $b(Y, Y)+b(W, W)$ and compare them. The computation shows that the claimed upper bound in (2.3) is, after dividing $\hat{\lambda}$ on both side, equivalent to

$$
\begin{aligned}
& (a-1) \cos ^{2} \theta+(a-1) \eta^{2} \sin ^{2} \theta+1+\eta^{2}-2(a-1) \eta \sin \theta \cos \theta \\
& \leq \frac{2 a}{a+1}\left(1+\eta^{2}+(a-1) \cos ^{2} \theta+\eta^{2}(a-1) \sin ^{2} \theta\right) .
\end{aligned}
$$

Rearranging and dividing by the positive number $(a-1)$ shows that this is equivalent to

$$
-2 \eta \sin \theta \cos \theta \leq \frac{1}{a+1}\left(\eta^{2}+1\right)+\frac{a-1}{a+1} \cos ^{2} \theta+\frac{a-1}{a+1} \eta^{2} \sin ^{2} \theta .
$$

This follows from

$$
\begin{aligned}
\left(1+\eta^{2}\right)+(a-1) \cos ^{2} \theta+\eta^{2}(a-1) \sin ^{2} \theta & \geq 2 \eta-2(a-1) \eta \cos \theta \sin \theta \\
& \geq-2(a+1) \eta \sin \theta \cos \theta .
\end{aligned}
$$

Clearly equality implies that $|\eta|=1$, namely $|Y|=|W|$.

The consequence of the above lemma is a generalization of Berger's well-known lemma, which we state below.

Corollary 2.2. Assume that $(M, g)$ has $\lambda$-pinched flag curvature with dimension $n \geq 4$. Assume that the sectional curvature is nonnegative at $x$ and $X, Y, Z, W \in T_{x} M$ are four vectors mutually orthogonal. Then

$6 \frac{1+\lambda}{1-\lambda}|R(X, Y, Z, W)| \leq k(X, Z)+k(Y, Z)+k(X, W)+k(Y, W)+2 k(X, Y)+2 k(Z, W)$. If equality holds and $\operatorname{Rm}(x) \neq 0$, then vectors $X, Y, Z, W$ have the same norm.

Proof. As before let $a=1 / \lambda$. The result follows from Corollary 2.1 and the estimate (2.3). In fact, applying (2.3) one can estimate

$$
\begin{aligned}
& R(Y+W, Z+X, Z+X, Y+W)-R(Y, Z+X, Z+X, Y)-R(W, Z+X, Z+X, W) \\
& \quad=R(Y, Z+X, Y, Z+X)+R(W, Z+X, W, Z+X)-R(Y+W, Z+X, Y+W, Z+X)
\end{aligned}
$$




$$
\geq\left(1-\frac{2 a}{a+1}\right)(R(Y, Z+X, Y, Z+X)+R(W, Z+X, W, Z+X)) .
$$

Applying the similar estimate to the other three groups in the expression of $R(X, Y, Z, W)$ from Corollary 2.1, the lower bound of $-R(X, Y, Z, W)$ follows by expanding $R(Y, Z+X, Y, Z+X)+R(W, Z+X, W, Z+X)$, etc., and regrouping them. The upper bound is similar. We leave the detailed checking to the readers.

REMARK 2.3. Berger's lemma concludes a similar estimate as that of Corollary 2.2 under the stronger assumption that the sectional curvature is $\lambda$-pinched.

Next we shall estimate the complex sectional curvature via the pinching constant $\lambda$.

Lemma 2.3. Assume that $(M, g)$ (with dimension $n \geq 4$ ) has nonnegative sectional curvature and satisfies (1.1) at $x \in M$. Then for any $X, Y, Z, W \in T_{x} M$ which are mutually orthogonal with $|X|=|Z|=1,1 \geq|Y|,|W| \geq 0$, we have the following estimates:

$$
R(U, V, \bar{U}, \bar{V}) \geq \begin{cases}\frac{2(4 \lambda-1)}{3}(k(X, \hat{Y})+k(Z, \hat{W})), & \text { for } \lambda \leq \frac{1}{4} \\ \frac{2(4 \lambda-1)}{3} \cdot \min \{k(X, \hat{Y}), k(Z, \hat{W})\}, & \text { for } \frac{1}{1+\sqrt{3}} \geq \lambda>\frac{1}{4} \\ \frac{2 \lambda(1+2 \lambda)}{3(1+\lambda)} \max \{k(X, \hat{Y}), k(Z, \hat{W})\}, & \text { for } 1>\lambda>\frac{1}{1+\sqrt{3}}\end{cases}
$$

Here $U=X+\sqrt{-1} Y, V=Z+\sqrt{-1} W, \hat{Y}$ and $\hat{W}$ are the unit vectors in the direction of $Y$ and $W$. The equality in the case $\lambda \leq 1 / 4$ can only happen when $|X|=|Y|=$ $|W|=|Z|$ and $\lambda k(X, Y)=\lambda k(Z, W)=k(X, Z)=k(X, W)=k(Y, Z)=k(Y, W)$, provided that the sectional (or scalar) curvature is positive.

Proof. Recall that

$$
R(U, V, \bar{U}, \bar{V})=k(X, Z)+k(X, W)+k(Y, Z)+k(Y, W)-2 R(X, Y, Z, W) .
$$

By Corollary 2.2 we have that

$$
\begin{aligned}
R(U, V, \bar{U}, \bar{V}) \geq \frac{2(a+2)}{3(a+1)}(k(X, Z)+k(X, W) & +k(Y, Z)+k(Y, W)) \\
& -\frac{2(a-1)}{3(a+1)}(k(X, Y)+k(Z, W)) .
\end{aligned}
$$

Here $a=1 / \lambda$ as before. We focus on $\lambda \leq 1 / 4$, namely $a \geq 4$ case. Now write $t=|Y|^{2}, \xi=|W|^{2}$ and $Y=t^{1 / 2} \hat{Y}, W=\xi^{1 / 2} \hat{W}$. Consider

$$
\begin{aligned}
Q(t, \xi)=(a+2)(k(X, Z)+k(X, \hat{W}) \xi+k(\hat{Y}, Z) t & +k(\hat{Y}, \hat{W}) t \xi) \\
& -(a-1)(k(X, \hat{Y}) t+k(Z, \hat{W}) \xi)
\end{aligned}
$$

as a function defined for $0 \leq \xi, t \leq 1$. Its minimum can only possibly be achieved for $t$ and $\xi$ taking values in $\{0,1\}$. For $t=1$ and $\xi=1$, we deduce that

$$
Q \geq \frac{(4-a)(a+1)}{a}(k(X, \hat{Y})+k(Z, \hat{W}))
$$

which implies the result. If $t=0, \xi=1$,

$$
Q \geq(a+2) k(X, Z)+\frac{2+2 a-a^{2}}{a} k(Z, \hat{W})
$$




$$
\geq \frac{(4-a)(a+1)}{a} k(Z, \hat{W}) .
$$

The case $t=1, \xi=0$, is symmetric to the one above with the estimate $Q \geq$ $\frac{(4-a)(a+1)}{a} k(X, \hat{Y})$. For $t=0, \xi=0$, we have that $Q \geq \frac{a+2}{a} k(X, \hat{Y})$. Putting them together we have the estimate for $\lambda \leq 1 / 4$. The two cases can be done similarly.

Proof of Theorem 1.1. Let $\mathrm{Rm} \in S_{B}^{2}(\mathfrak{s o}(n))$ be an algebraic curvature operator with quarter-pinched flag curvature. We want to check that the curvature of $\mathrm{Rm}$ evaluated at a complex plane $\sigma \subset \mathbb{C}^{n}$ is nonnegative. Next we choose a good basis of $\sigma$ as follows. We endow $\mathbb{C}^{n}$ with the usual scalar product $\langle\langle x, y\rangle\rangle=\bar{y}^{t r} x$. Let $U$ be a unit vector in $\sigma$ which maximizes among all unit vectors the real part of the scalar product $\langle\langle U, \bar{U}\rangle\rangle$. Let $V$ be a unit vector in $\sigma$ perpendicular to $U$ such that $\langle\langle V, \bar{V}\rangle\rangle$ is a nonnegative real number. By the choice of $U$ we have $\langle\langle U, V\rangle\rangle=\langle\langle\bar{U}, V\rangle\rangle=0$. Moreover, $\langle\langle U, \bar{U}\rangle\rangle$ and $\langle\langle V, \bar{V}\rangle\rangle$ are real. Let $X, Y, Z, W \in \mathbb{R}^{n}$ be such that $U=$ $X+\sqrt{-1} Y, V=Z+\sqrt{-1} W$. It is straightforward to check that $X, Y, Z, W$ are pairwise orthogonal. Furthermore $|X| \geq|Y|$ and $|Z| \geq|W|$.

Finally we rescale $U$ and $V$ by a real number such that $|X|=|Z|=1$. The nonnegativity now follows from the previous lemma.

The above argument also proves that a curvature operator $\mathrm{Rm} \neq 0$ with nonnegative sectional curvature, $\lambda$-pinched flag curvature has positive complex sectional curvature if $\lambda>1 / 4$. Tracing the proof it is easy to see the following corollary.

Corollary 2.4. Let $(M, g)$ be as in Theorem 1.1. Then if $R(U, V, \bar{U}, \bar{V})=0$ for a pair of vectors $U, V$ spanning a complex plane $\sigma$. Then $\sigma$ is isotopic. Namely for any $T \in \sigma,\langle T, T\rangle=0$.

\section{Consequences}

Using the work of [BöW2] and [BrS1], Theorem 1.1 has a more general differential sphere theorem as its direct consequence. Recall that it was proved in [BrS1] that

Theorem 3.1 (Brendle-Schoen). The Ricci flow on a compact manifold preserves the cones consisting of

(i) the curvature operators with nonnegative complex sectional curvature; and

(ii) the curvature operators with positive complex sectional curvature.

Originally in $[\mathrm{BrS} 1]$, the authors proved that the condition that $M \times \mathbb{R}^{2}$ has nonnegative isotropic curvature is preserved under Ricci flow. However it turns out (cf. $[\mathrm{NiW}]$ ) that the condition $M \times \mathbb{R}^{2}$ has nonnegative isotropic curvature is equivalent to $M$ has nonnegative complex sectional curvature. There exists a more direct proof of Brendle-Schoen's above result in [NiW] using complex numbers.

Using the above result, Brendle and Schoen further proved that any compact manifold with strictly 1/4-pinched sectional curvature must be a diffeomorphic sphere, via the following general invariant cone construction of [BöW2] by Böhm and the second author, and their consequence on the convergence of the Ricci flow. (Cf. Theorem 6.2 of $[\mathrm{W}]$. 
Theorem 3.2 (Böhm-Wilking). Let $C$ be an $\mathrm{O}(n)$-invariant convex cone of full dimension in the vector space of algebraic curvature operators $S_{B}^{2}(\mathfrak{s o}(n))$ with the following properties:

(i) $C$ is invariant under the ODE $\frac{d \mathrm{Rm}}{d t}=\mathrm{Rm}^{2}+\mathrm{Rm}^{\#}$.

(ii) $C$ contains the cone of nonnegative curvature operators, or slightly weaker all nonnegative curvature operators of rank 1.

(iii) $C$ is contained in the cone of curvature operators with nonnegative sectional curvature.

Then for any compact manifold $(M, g)$ whose curvature operator is contained in the interior of $C$ at every point $p \in M$, the normalized Ricci flow evolves $g$ to a limit metric of constant sectional curvature.

Proof. It follows from the proof of Lemma 3.4 and 3.5 of [BöW2] verbatim.

Combining Theorem 3.1 and Theorem 3.2 we have that if $(M, g)$ is a Riemannian manifold with positive sectional curvature such that (1.1) holds for some $\lambda>1 / 4$, then $(M, g)$ is diffeomorphic to a spherical space form. In fact one can arrive at the same conclusion assuming only that $(M, g)$ is a nonnegatively curved manifold with quarter-pinched flag curvature and with some point $x$ at which the curvature operator $\operatorname{Rm}(x) \neq 0$ has strictly quarter-pinched flag curvature.

In [BrS2], the manifolds with weakly 1/4-pinched sectional curvature were analyzed. Since the key step is a result on the rigidity of manifolds with nonnegative complex sectional curvature (Theorem 2 of [BrS2]), which in turn relies on a general strong maximum principle result, one can modify this part to obtain the generalization stated in Corollary 1.2.

Proof of Corollary 1.2. By [BrS2], particularly, Proposition 11 therein on the rigidity of manifolds with nonnegative complex sectional curvature, one can reduce the proof of Corollary 1.2 to show that if $M$ is a Kähler manifold with 1/4-pinched flag curvature, then the universal cover of $M$ is isometric to $\mathbb{C} P^{m}(n=2 m)$. Now consider the complex sectional curvature $R(U, V, \bar{U}, \bar{V})$ for any $U, V \in T^{1,0} M$ with $|U|=|V|$ and $\langle U, \bar{V}\rangle=0$. By the Kählerity it must be zero. Write as before that $U=X+\sqrt{-1} Y$ and $V=Z+\sqrt{-1} W$. We deduce that $Y=-J X, W=-J Z$, By tracing the equality case in section 2 , we have $k(X, J X)=k(Z, J Z)=4 k(X, Z)=$ $4 k(X, W)=4 k(Y, Z)=4 k(Y, W)$. This proves the result for the case $m \geq 3$ since for any $U_{1}, U_{2} \in T^{1,0} M$ one can find $U_{3}$ such that $\left\langle U_{3}, \bar{U}_{i}\right\rangle=0$ for $i=1,2$. Hence $M$ has constant holomorphic sectional curvature at any given point $p \in M$. By the Kählerian analogue of Schur's lemma we can conclude that $M$ is of constant holomorphic sectional curvature. For $m=2$, the result has been proved in [AnN]. (See also the next section for a different argument on this part.)

\section{An Example and the Dual Case}

In this section, first for $n \geq 4$, we present an example of algebraic curvature operator which has 1/4-pinched sectional curvature, but only 1/2-pinched flag curvature (by 
scaling, one can easily derive examples which has $\lambda^{2}$-pinched sectional curvature, but only $\lambda$-pinched flag curvature).

Define an algebraic curvature operator on $\mathbb{R}^{4}$ by

$$
\begin{aligned}
\mathrm{Rm}=4\left(e_{1} \wedge e_{2}\right) \otimes\left(e_{1} \wedge e_{2}\right)+ & 2\left(e_{1} \wedge e_{3}\right) \otimes\left(e_{1} \wedge e_{3}\right) \\
+ & 2\left(e_{1} \wedge e_{4}\right) \otimes\left(e_{1} \wedge e_{4}\right)+2\left(e_{2} \wedge e_{3}\right) \otimes\left(e_{2} \wedge e_{3}\right) \\
& +2\left(e_{2} \wedge e_{4}\right) \otimes\left(e_{2} \wedge e_{4}\right)+\left(e_{3} \wedge e_{4}\right) \otimes\left(e_{3} \wedge e_{4}\right) .
\end{aligned}
$$

Here $\left\{e_{i}\right\}$ is a orthonormal frame. First one can easily check that $\mathrm{Rm}$ is an algebraic curvature operator since it is a symmetric tensor of $\wedge^{2}\left(\mathbb{R}^{4}\right)$ and satisfies the first Bianchi identity. It is easy to see that the sectional curvature of $\mathrm{Rm}$ is $1 / 4$-pinched. On the other hand, for the flag curvature it is only 1/2-pinched. It is clear that the eigenvalues of $R_{e}(\cdot, \cdot)$ are 1/2-pinched, when $e=e_{i}$. To check for generic vector it is sufficient to check for $e=\cos \theta e_{1}+\sin \theta e_{3}$ for any $\theta \in \mathbb{R}$. Direct computation verifies that the three eigenvectors of $R_{e}(\cdot, \cdot)$ are $e_{2}, e_{4}$ and $-\sin \theta e_{1}+\cos \theta e_{3}$ with eigenvalues $2 \cos ^{2} \theta+2,1+\cos ^{2} \theta$ and 2 correspondingly. They have pinching constant $1 / 2$ again. The example shows that if an algebraic curvature tensor $R$ has $\lambda$-pinched flag curvature, its sectional curvature has pinching constant at the best $\lambda^{2}$. In particular, if Rm has 1/4-pinched flag curvature, one at best can expect its sectional curvature is $1 / 16$-pinched. This example can easily be generalized to any dimensions by replacing $e_{3}$ and $e_{4}$ by $e_{i}$ for $n \geq i \geq 3$.

Finally we observe that the discussions in section 2 can be adapted to the manifolds with nonpositive sectional curvature and 1/4-pinched flag curvature. Note that in the case that $(M, h)$ has nonpositive sectional curvature we say that it has $\lambda$-pinched flag curvature if and only if

$$
\lambda R_{e}(X, X) \geq R_{e}(Y, Y)
$$

holds for any $e \neq 0$, and any $X, Y$ in the orthogonal complement of $e$ with $|X|=$ $|Y|=1$. Clearly this is equivalent to saying that the curvature tensor $R^{\prime}=-R$ has nonnegative sectional curvature with $\lambda$-pinched flag curvature. The following is the analogue of Theorem 1.1

Corollary 4.1. Assume that $\left(M^{n}, g\right)$ is a Riemannian manifold $(n \geq 4)$ having nonpositive sectional curvature. If (4.1) holds for some $\lambda \geq 1 / 4$, then $(M, g)$ has nonpositive complex sectional curvature. Moreover if the sectional curvature is negative at $x$ and (4.1) holds at $x$ with $\lambda>1 / 4$, for all $e(x) \neq 0$, then there exists $\epsilon(x)>0$, depending on $\lambda(x)$ and $\operatorname{Scal}(x)$ such that at $x,(\mathrm{Rm}+\epsilon \mathrm{I})$ has nonpositive complex sectional curvature, where I is the identity of $S^{2}\left(\wedge^{2}(\mathbb{R})\right)$. In particular $(M, g)$ has negative complex sectional curvature at $x$.

Now Theorem 1.3 follows from the existence result of Eells and Sampson on the harmonic maps and the following general rigidity theorem.

Theorem 4.2. Let $X^{m}(m \geq 2)$ be a compact Kähler manifold of complex dimension $m, M^{n}$ be a Riemannian manifold of even dimension (real) $n=2 m$ with nonpositive sectional curvature and satisfying (4.1) with $\lambda=1 / 4$. Suppose that there is continuous map $f_{0}: X \rightarrow M$ of nonzero degree. Then $M$ is a locally symmetric space with universal cover $\mathbb{B}^{m}(n=2 m)$. 
Proof. Here we follow the line of argument in [YZ]. First by the existence theorem of Eells and Sampson $f_{0}$ is homotopic to a harmonic map $f$ which is onto. Let $\mathcal{Z}$ be the critical value of $f$. First restrict the discussion on $M \backslash \mathcal{Z}$. Endow $M \backslash \mathcal{Z}$ with an almost complex structure (still denoted by $J$ ) by pushing forward the complex $J$ on $X$ via $f_{*}$. Let $\left(z_{1}, \ldots, z_{m}\right)$ be a local complex coordinate of $X$. Let $U_{i}=f_{*}\left(\partial / \partial z_{i}\right)$. Since by Corollary $4.1 M$ has nonpositive complex sectional curvature, a result of Sampson, Theorem 1 of $[\mathrm{S}]$, asserts that

$$
\nabla_{U_{i}} \bar{U}_{j}=0, \quad R\left(U_{i}, U_{j}, \bar{U}_{i}, \bar{U}_{j}\right)=0 .
$$

This particularly implies that $R(U, V, \bar{U}, \bar{V})=0$ for any $U, V \in T^{1,0} M$ (here the decomposition is with respect to the push-forward complex structure). The dual version of Corollary 2.4 asserts that $\sigma=\operatorname{Span}\{U, V\}$ is isotropic, if it is a complex plane. This in turn implies that $\langle U, U\rangle=0$, hence the metric $h$, with respect to the push-forward of the complex structure, is Hermitian. Using $\nabla_{U_{i}} \bar{U}_{j}=0$, one can check, via a direct computation, that the Kähler form of $(M \backslash \mathcal{Z}, h, J)$ is closed (this is the observation of [YZ], the proof of Theorem 3). The Kählerity conclusion is first made on $M \backslash \mathcal{Z}$ and then extends to $M$ since $M \backslash \mathcal{Z}$ is open and dense. Once we know that $(M, h, J)$ is a Kähler manifold with 1/4-pinched flag curvature, when $m \geq 3$, the claimed result follows similarly as in the proof of Corollary 1.2. More precisely, we can show, in exactly the same manner as in Corollary 1.2 that, at every point $x \in M$, its holomorphic sectional curvature is independent of the choice of the complex lines. Hence the result follows by the holomorphic Schur lemma. When $m=2$, the last conclusion can be seen by observing that at every point $x \in M$, the curvature operator $\mathrm{Rm}$ is the multiple of curvature operator of $\mathbb{B}^{2}$. The reason is that the condition of $1 / 4$-flag curvature pinching is a convex $\mathrm{U}(2)$ invariant condition including the curvature operator of $\mathbb{B}^{2}$. On the other hand, one can check easily that there exist some small perturbations towards the holomorphic Weyl part, as well as the traceless Ricci part, which violates the condition of the flag curvature being $1 / 4$-pinched. This shows that the curvature operator of $\mathbb{B}^{2}$ is the only curvature operator of Kähler manifolds satisfying the flag curvature 1/4-pinching condition.

REMARK 4.3. It is very reasonable to expect that a similar result as Theorem 4.2 holds for quaternion-Kähler manifold with nonpositive 1/4-pinched flag curvature.

\section{Odd Dimensions - A Vanishing Theorem}

As another application of the estimates from section 2 we prove a generalization of an earlier result of Berger (Theorem 2 of [B1]) on odd-dimensional manifolds. Again, we relax the assumption on the pointwise $\lambda$-pinching of the sectional curvature to the $\lambda$-pinched flag curvature. The difference on the assumption does cause subtlety which needs to be dealt with more carefully.

Theorem 5.1. Let $(M, g)$ be a nonnegatively curved compact Riemannian manifold of odd-dimension $n$. Assume that it has $\lambda$-pinched flag curvature with $\lambda \geq \frac{n-3}{4 n-9}$ and its scalar curvature satisfying $\operatorname{Scal}(x)>0$ for some point $x \in M$. Then the second Betti number of $M$ vanishes. 
Proof. Let $n=2 m+1$. As in [B1] the proof is via the Bochner-Weitzenböck formula,

$$
\Delta_{d}=-\operatorname{tr} \nabla^{2}+\sum_{i, j} \omega_{i} \wedge i\left(E_{j}\right) \mathcal{R}_{E_{i} E_{j}},
$$

on harmonic 2-forms. Here $E_{i}$ is a orthonormal frame, $\mathcal{R}$ is the curvature of the induced covariant differentiation on two forms, $i(\cdot)$ is the contraction operator. We first prove the result for the case that $\lambda>\frac{2(m-1)}{8 m-5}$. By Hodge theory, it suffices to show that any harmonic 2 -form is trivial. With some calculations one can show that for any two form $\Omega$

$$
\sum_{i, j} \omega_{i} \wedge i\left(E_{j}\right) \mathcal{R}_{E_{i} E_{j}} \Omega=(\operatorname{Ric} \wedge \mathrm{id}-\operatorname{Rm})(\Omega) .
$$

(Here $A \wedge B(X \wedge Y) \doteqdot \frac{1}{2}(A(X) \wedge B(Y)+B(X) \wedge A(Y))$.) The key is to show that if $\lambda>\frac{2(m-1)}{8 m-5}$, then $\operatorname{Ric} \wedge \mathrm{id}-\mathrm{Rm}>0$ as a symmetric tensor on $\wedge^{2}\left(\mathbb{R}^{n}\right)$ (which can be identified with $\mathfrak{s o}(n))$. Also observe an interesting fact that by Lemma 2.1 of [BöW2], Ric $\wedge$ id $-\mathrm{Rm}=\mathrm{Rm} \# \mathrm{I}$, even though we do not make use of it in our proof. Here id $: \mathbb{R}^{n} \rightarrow \mathbb{R}^{n}, \mathrm{I}: S_{B}^{2}(\mathfrak{s o}(n)) \rightarrow S_{B}^{2}(\mathfrak{s o}(n))$ are two identity maps.

Given $\Omega \in \wedge^{2}\left(\mathbb{R}^{n}\right)$, by linear algebra one can find orthonormal frame $X_{1}, X_{2}, \ldots, X_{2 m+1}$ such that

$$
\Omega=\sum_{i=1}^{m} \alpha_{i} X_{2 i-1} \wedge X_{2 i}
$$

Computation shows that

$$
\begin{aligned}
& 2\langle(\operatorname{Ric} \wedge \mathrm{id}-\operatorname{Rm})(\Omega), \Omega\rangle=\sum_{1 \leq j \leq m, 1 \leq s \leq n} \alpha_{j}^{2}(k(2 j-1, s)+k(2 j, s)) \\
& -2 \sum_{1 \leq i, j \leq m} \alpha_{i} \alpha_{j} R\left(X_{2 i-1}, X_{2 i}, X_{2 j-1}, X_{2 j}\right) .
\end{aligned}
$$

Here $k(2 j, s) \doteqdot k\left(X_{2 j}, X_{s}\right)$. Writing the last term above as

$$
2 \sum_{1 \leq j \leq m} \alpha_{j}^{2} k(2 j-1,2 j)+2 \sum_{1 \leq i \neq j \leq m} \alpha_{i} \alpha_{j} R\left(X_{2 i-1}, X_{2 i}, X_{2 j-1}, X_{2 j}\right)
$$

and combining the similar terms we arrive at

$$
\begin{aligned}
& 2\langle(\operatorname{Ric} \wedge \mathrm{id}-\operatorname{Rm})(\Omega), \Omega\rangle \\
& =\frac{1}{2} \sum_{i \neq j}\left(\alpha_{i}^{2}+\alpha_{j}^{2}\right)(k(2 j-1,2 i)+k(2 j-1,2 i-1)+k(2 j, 2 i)+k(2 j, 2 i-1)) \\
& \quad+\sum_{1 \leq j \leq m} \alpha_{j}^{2}(k(2 j-1, n)+k(2 j, n)) \\
& \quad-2 \sum_{1 \leq i \neq j \leq m} \alpha_{i} \alpha_{j} R\left(X_{2 i-1}, X_{2 i}, X_{2 j-1}, X_{2 j}\right) .
\end{aligned}
$$

Here $\sum_{i \neq j}$ means $\sum_{1 \leq i \neq j \leq m}$. Now we use Corollary 2.2 to estimate the last term. Let

$$
\beta=\frac{3(1+\lambda) \lambda}{2(1-\lambda)(m-1)}
$$


and write

$$
\begin{aligned}
2\left|\alpha_{i}\right|\left|\alpha_{j}\right| R\left(X_{2 i-1}, X_{2 i},\right. & \left.X_{2 j-1}, X_{2 j}\right)=2(1-\beta)\left|\alpha_{i}\right|\left|\alpha_{j}\right| R\left(X_{2 i-1}, X_{2 i}, X_{2 j-1}, X_{2 j}\right) \\
& +2 \beta R\left(\left|\alpha_{i}\right|^{1 / 2} X_{2 i-1},\left|\alpha_{i}\right|^{1 / 2} X_{2 i},\left|\alpha_{j}\right|^{1 / 2} X_{2 j-1},\left|\alpha_{j}\right|^{1 / 2} X_{2 j}\right) .
\end{aligned}
$$

Now via Corollary 2.2 the right-hand side above can be estimated by

$$
\begin{gathered}
\frac{1-\lambda}{3(1+\lambda)}\left|\alpha_{i}\right|\left|\alpha_{j}\right|(k(2 i, 2 j-1)+k(2 i, 2 j)+k(2 i-1,2 j-1)+k(2 i-1,2 j)) \\
+\frac{2(1-\lambda)}{3(1+\lambda)}(1-\beta)\left|\alpha_{i}\right|\left|\alpha_{j}\right|(k(2 i, 2 i-1)+k(2 j, 2 j-1)) \\
+\frac{2(1-\lambda)}{3(1+\lambda)} \beta\left(k(2 i, 2 i-1) \alpha_{i}^{2}+k(2 j, 2 j-1) \alpha_{j}^{2}\right) .
\end{gathered}
$$

Observe that

$\sum_{1 \leq j \leq m} \alpha_{j}^{2}(k(2 j-1, n)+k(2 j, n))-\sum_{i \neq j} \frac{2(1-\lambda)}{3(1+\lambda)} \beta\left(k(2 i, 2 i-1) \alpha_{i}^{2}+k(2 j, 2 j-1) \alpha_{j}^{2}\right) \geq 0$

by the choice of $\beta$ and the $\lambda$-pinching of the flag curvature. Putting the above estimates together we have that

$$
\begin{aligned}
& 2\langle(\operatorname{Ric} \wedge \mathrm{id}-\operatorname{Rm})(\Omega), \Omega\rangle \\
& \geq\left(1-\frac{1-\lambda}{3(1+\lambda)}\right) \sum_{i \neq j} \frac{\alpha_{i}^{2}+\alpha_{j}^{2}}{2}(k(2 j-1,2 i)+k(2 j-1,2 i-1)+k(2 j, 2 i)+k(2 j, 2 i-1)) \\
& \quad-\frac{2(1-\lambda)}{3(1+\lambda)}(1-\beta) \sum_{i \neq j}\left|\alpha_{i}\right|\left|\alpha_{j}\right|(k(2 i, 2 i-1)+k(2 j, 2 j-1)) .
\end{aligned}
$$

Also observe that $k(2 i, 2 i-1) \leq \frac{1}{\lambda}(k(2 j, 2 i)+k(2 j-1,2 i))$ and $2 k(2 j, 2 j-1) \leq$ $\frac{1}{\lambda}(k(2 j-1,2 i-1)+k(2 j, 2 i-1))$. The positivity of $\langle(\operatorname{Ric} \wedge \mathrm{id}-\operatorname{Rm})(\Omega), \Omega\rangle$ holds if

$$
\left(1-\frac{1-\lambda}{3(1+\lambda)}\right) \lambda>\frac{1-\lambda}{3(1+\lambda)}(1-\beta)
$$

which, after plugging in the expression of $\beta$, is equivalent to

$$
(8 m-5) \lambda^{2}+(6 m-3) \lambda-2(m-1)>0 .
$$

It is a simple matter to check that if $\lambda>\frac{2(m-1)}{8 m-5}$ the above inequality holds up. This completes the proof of the theorem for $\lambda>\frac{2(m-1)}{8 m-5}$.

For the case that $\lambda \geq \frac{2(m-1)}{8 m-5}\left(=\frac{n-3}{4 n-9}\right)$, observe that the above argument shows that $\Omega$ is parallel (after integration by parts on the manifold). On the other hand, since the manifold has positive sectional curvature at some point and it is of odd dimension its holonomy group has to be $\mathrm{SO}(n)$. The fact that $\Omega$ is parallel implies that as an element in $\mathfrak{s o}(n)$ it is fixed by the conjugate action of $\mathrm{SO}(n)$. This is impossible unless $\Omega=0$.

Corollary 1.4 is a simple consequence of the vanishing of the second cohomology and the fact that a positively curved compact manifold has finite fundamental group. We are also aware of the fact that in the case of sectional curvature pinching, Berger did push the constant further down to $4 / 23$ for 5 -manifolds [B2]. 
REMARK 5.2. From the proof it is easy to see that the nonnegativity of the complex sectional curvature implies the nonnegativity of Bochner-Weizenböck curvature on two forms, namely Ric $\wedge \mathrm{id}-\mathrm{Rm} \geq 0$. The argument in the proof of Theorem 5.1 also proves that if $M$ is an even dimensional manifold with positive isotropic curvature, then the second Betti number $b_{2}(M)=0$, a result of Micallef and Wang [MW]. Moreover, it proves the same result for an odd-dimensional manifold $\left(M^{n}, g\right)$ $(n=2 m+1)$ with positive isotropic curvature and 2-nonnegative sectional curvature (namely $k(1 ; i)+k(1 ; j) \geq 0$ for $2 \leq i \neq j \leq n$ ), which covers the case of $M=\mathbb{S}^{2 m} \times \mathbb{S}^{1}$.

\section{Pinching Theorems Below One Quarter}

First we remark that Corollary 1.2 carries over to the orbifolds (due to Theorem 3.2 in section 3 and Proposition 5.2 of [BöW2]).

Theorem 6.1. In dimensions above 2 a compact Riemannian orbifold with 1/4pinched flag curvature either admits a metric of constant sectional curvature or it is isometric to the quotient of a rank 1 symmetric space by a finite group action.

This generalization is interesting since in even dimensions the situation changes dramatically if one relaxes the assumption:

Proposition 6.1. In each even dimension $2 n>2$ and for each $\lambda<1 / 4$ there is a compact Riemannian orbifold with sectional curvature $\lambda<K<1$ which is not given as the quotient of a manifold by a finite group action.

Proof. Consider on $\mathbb{S}^{2 n+1}$ (viewed as $\left(a_{1}, \ldots, a_{n+1}\right) \in \mathbb{C}^{n+1}$ with $\sum_{i=1}^{n+1}\left|a_{i}\right|^{2}=1$ ), the $\mathrm{S}^{1}$-action given by $z\left(a_{1}, \ldots, a_{n+1}\right)=\left(z^{k+1} a_{1}, z^{k} a_{2}, \ldots, z^{k} a_{n+1}\right)$. The quotient $M_{k} \doteqdot \mathbb{S}^{2 n+1} / \mathrm{S}^{1}$ is an orbifold - a weighted complex projective space. If we endow $M_{k}$ with the induced metric it is straightforward to check that as $k \rightarrow \infty$ the lower and upper curvature bounds of $M_{k}$ converge to the lower and upper curvature bounds of $\mathbb{C} P^{n}$.

On the other hand $M_{k}$ is not covered by a manifold. In fact, consider the frame bundle $F$ corresponding to the horizontal distribution on $\mathbb{S}^{2 n+1}$. The action of $\mathbf{S}^{1}$ induces a free action on $F$ and the quotient $\bar{F}=F / S^{1}$ can be naturally identified with the frame bundle of the orbifold $M_{k}$. Thus the fundamental group of the frame bundle of $M_{k}$ has at most two elements and $M_{k}$ is not a finite quotient of a manifold.

The above proposition shows that in general the classification of nearly onequarter-pinched manifolds of $[\mathrm{PT}]$ cannot be easily adapted to orbifolds. (But also see Theorem 6.4.) In odd dimensions the situation is quite different and we do have the following generalization.

Theorem 6.2. For each constant $C$ and each odd dimension $n$ there is an $\varepsilon>0$ such that the following holds. Let $\left(M^{n}, g\right)$ be a nonnegatively curved Riemannian orbifold with $\frac{1-\varepsilon}{4}$-pinched flag curvature and scalar curvature satisfying $1 \leq \mathrm{Scal} \leq C$. Then $M$ admits a metric of constant curvature. 
Proof. First notice that the assumptions imply the existence of positive constants $c_{1}, c_{2}$ such that the sectional curvature $K$ of $M$ is globally pinched with $c_{1} \leq K \leq c_{2}$. In order to avoid too much technical difficulties we will frequently replace the orbifold $M$ by the frame bundle $F$ of the orbifold. Recall that $F$ is a manifold which can be endowed with a natural connection metric.

The next step is to show that the universal cover $\tilde{F}$ of the frame bundle $F$ of the orbifold $M$ has finite second homology.

We may assume that $\frac{1-\varepsilon}{4}>\frac{n-3}{4 n-9}$. By Theorem 5.1 this implies that the BochnerWeitzenböck operator of $M$ on two forms (namely Rm \# I) is positive. We only need to check that the frame bundle $F$ has a metric such that the Bochner-Weitzenböck operator on two forms is positive. We endow $F$ with the connection metric and shrink the fiber $\mathrm{SO}(n)$ by a small factor $\lambda$. Let $g$ denote the bi-invariant metric on $\mathrm{SO}(n)$. It is easy to see that the difference of the curvature tensor of $\left(F, g_{\lambda}\right)$ and the curvature tensor of the product $M \times\left(\mathrm{SO}(n), \lambda^{2} g\right)$ converges to zero. Since the Bochner-Weitzenböck operators of $M$ and $\mathrm{SO}(n)$ are positive it is easy to see that the smallest eigenvalue of the Bochner-Weitzenböck operator of $M \times\left(\mathrm{SO}(n), \lambda^{2} g\right)$ increases if $\lambda$ decreases. This shows that $F$ and its universal cover have finite second homology, by Theorem 5.1.

Next we run the Ricci flow on $M$. We want to apply a dynamical version of the maximum principle [ChL], [BöW1]. We assume $\varepsilon<1 / 2$. For a large constant $C_{2}$ and $C_{3}>>C_{2}$ and $t \in\left[0,1 / C_{3}^{2}\right]$ we define a set $S(t) \subset S_{B}^{2}(\mathfrak{s o}(n))$ as follows. Let $S(t)$ be the set of all algebraic curvature operators which have $\frac{1-\varepsilon-C_{3} t}{4}$-pinched flag curvature and whose scalar curvature satisfies $1 \leq$ Scal $\leq C+C_{2} t$. Furthermore we require that for any $\mathrm{Rm} \in S(t)$ the curvature operator $\left(\mathrm{Rm}+C_{3} \varepsilon \cdot e^{C_{3}^{2} t} \mathrm{I}\right)$ has nonnegative complex sectional curvature.

It is straightforward to check that one can choose $C_{2}$ and $C_{3}$ independent of $\varepsilon$ such that the family $S(t)$ is invariant under the Ricci flow ODE,

$$
\frac{d}{d t} \mathrm{Rm}=2\left(\mathrm{Rm}^{2}+\mathrm{Rm}^{\#}\right),
$$

that is if $\operatorname{Rm}(t)$ is a solution to the ODE and $\operatorname{Rm}\left(t^{\prime}\right) \in S\left(t^{\prime}\right)$, then $\operatorname{Rm}(t) \in S(t)$ for $t \in\left[t^{\prime}, t_{0}\right]$. For example by ODE it is easy to see that there exists $t_{1}=t_{1}(C, n)$ such that $|\operatorname{Rm}| \leq C^{\prime}(C)$ on $\left[0, t_{1}\right]$ for some $C^{\prime}$ depending only on $C$ and dimension $n$. This gives an upper bound $C^{\prime \prime}(C)$ on the slope $\left|\frac{d}{d t} \mathrm{Rm}\right|$. From this it follows easily that $1 \leq$ Scal $\leq C+C_{2} t$ is preserved. Similarly one can show that $\frac{1-\varepsilon-C_{3} t}{4}$-pinched flag curvature is preserved. To see that the condition on almost nonnegative complex sectional curvature is preserved one has to use the fact that the nonnegative complex sectional curvature is an invariant condition and the that the ODE is locally Lipschitz. More precisely, by [BrS1] (as well as $[\mathrm{NiW}])$, if $\widehat{\mathrm{Rm}}=\mathrm{Rm}+C_{3} \varepsilon \cdot e^{C_{3}^{2} t}$ I has nonnegative complex sectional curvature, then $\widehat{\mathrm{Rm}}^{2}+\widehat{\mathrm{Rm}}^{\#}$ is contained in the tangent cone of complex nonnegative curvature operators. Since $\frac{d}{d t} \widehat{\mathrm{Rm}}=\mathrm{Rm}^{2}+\mathrm{Rm}^{\#}+C_{3}^{3} \varepsilon \cdot e^{C_{3}^{2} t} \mathrm{I}$ is greater than $\widehat{\mathrm{Rm}}^{2}+\widehat{\mathrm{Rm}}^{\#}$ for large $C_{3}$, the same holds for $\frac{d}{d t} \widehat{\mathrm{Rm}}$. By the dynamical version of Hamilton's 
maximum principle (see, for example, section 1 of [BöW1]) it follows that the Ricci flow on $M$ exists up to the time $t_{0}$ and $\left(M, g_{t}\right)$ satisfies the curvature condition $S(t)$.

We shall prove more generally that there exists a constant $\varepsilon$ such that the normalized Ricci flow evolves to a constant curvature limit metric. We argue by contradiction and consider a sequence of orbifolds $\left(M_{k}, g_{k}\right)$ with $\frac{1-\varepsilon_{k}}{4}$-flag pinching, scalar curvature $1 \leq$ Scal $\leq C$ and $\varepsilon_{k} \rightarrow 0$ such that for each element in the sequence the normalized Ricci flow does not converge to a constant curvature limit metric.

Without loss of generality we assume that $\left(M_{k}, g_{k}\right)$ cannot be written as a nontrivial quotient of another orbifold, since otherwise we may replace $M_{k}$ by its finite cover. This in turn implies that the fundamental group of the frame bundle $F_{k}$ of $\left(M_{k}, g_{k}\right)$ has at most two elements.

We next want to rule out collapsing. For each fixed $t \in\left[t_{0} / 4, t_{0}\right]$ we can use Shi's estimate to see that $\left(M_{k}, g_{k}(t)\right)$ has a priori bounds on all derivatives of the curvature tensor. We now look at the frame bundle $F_{k}$ of $\left(M_{k}, g_{k}(t)\right)$ with the induced connection metric. We now rescale the fibers of the frame bundle by a small factor $\lambda$ independent of $k$, such that there are constants $d_{1}, d_{2}>0$ for which the sectional curvature of this metric on $F_{k}$ is bounded above by $d_{2}$ and the Ricci curvature is bounded below by $d_{1}$.

Since $F_{k}$ has finite second homology we can employ one of the main theorems of $[\mathrm{PeT}]$ (see also Theorem 0.2 of $[\mathrm{FR}]$ ) which asserts that the injectivity radius of $F_{k}$ is bounded from below by a priori constant depending only on $d_{1}$ and $d_{2}$. Now by the compactness theorem (cf. [H3], noting that we do not need the compactness result of $[\mathrm{L}]$ on orbifolds), after passing to a subsequence we obtain a limit manifold $F$ endowed with a smooth family of metrics $g(t)$ with $t \in\left[t_{0} / 4, t_{0}\right]$ and with almost free isometric $\mathrm{SO}(n)$-action. The quotient orbifolds $(M, \bar{g}(t))=(F, g(t)) / \mathrm{SO}(n)$ have nonnegative complex sectional curvature, positive sectional curvature and $\bar{g}(t)$ is a solution to the Ricci flow.

The strong maximum principle of [BrS2] implies that either the complex sectional curvature is positive for $t>t_{0} / 4$ or the orbifold has nongeneric holonomy. Since the sectional curvature is positive and the orbifold is odd dimensional, the holonomy is generic and it follows that $(M, \bar{g}(t))$ has positive complex sectional curvature for $t>t_{0} / 4$. This in turn implies that $\left(M, g_{k}\left(t_{0} / 2\right)\right)$ has positive complex sectional curvature for infinitely many $k$. This is a contradiction since it implies that the normalized Ricci flow converges to a constant curvature limit metric for this infinite subsequence.

REMARK 6.3. (a) The above proof does not give effective bounds on $\varepsilon$. There is on the other hand a different proof which does give effective bounds. This alternative proof uses that the Ricci flow ODE behaves somewhat better in odd dimensions than in even dimensions. In fact with a bit of work one can show that for an algebraic nonvanishing curvature operator $R \in S_{B}^{2}(\mathfrak{s o}(2 n+1))$ with quarter-pinched flag curvature there exists an $\varepsilon>0$ such that $R+t\left(R^{2}+R^{\#}\right)$ has positive complex sectional curvature for all $t \in(0, \varepsilon]$. Knowing this fact one can just apply a dynamical version of the maximum principle to see that for nearly $1 / 4$-pinched operators, the 
Ricci flow ODE pinches towards positive complex sectional curvature. It would be interesting to see if one can modify this approach such that $\varepsilon$ becomes independent of $C$.

(b) Recall that by a theorem of Abresch and Meyer [AM], any simply connected odd-dimensional manifold with sectional curvature $K$ satisfying $\frac{1}{4\left(1+10^{-6}\right)^{2}} \leq K \leq 1$ is homeomorphic to a sphere. An obvious question arises whether or not one can improve the conclusion to diffeomorphism.

(c) For even-dimensional manifolds, since the collapsing cannot happen, the conclusion of Petersen and Tao's result still holds under the assumption of Theorem 6.2. On the other hand, the argument of the proof above can be sharpen to show that the weighted complex projective spaces are the only exceptions to a similar statement for even-dimensional orbifolds. See the theorem below.

The following theorem shows that the examples constructed in the proof of Proposition 6.1 are essentially the only additional examples that occur in even dimensions.

Theorem 6.4. Given $n$ and $C$ there is an $\varepsilon>0$ such that any Riemannian orbifold with $\frac{1-\varepsilon}{4}$-flag pinching and scalar curvature satisfying $1 \leq$ Scal $\leq C$ is either diffeomorphic to the quotient of rank-one symmetric space by a finite isometric group action or it is diffeomorphic to the quotient of a weighted complex projective space by a finite group action.

The proof requires the following lemma which can be proved along the same lines as in the proof of Theorem 1.3 (more precisely, the last part of the proof of Theorem 4.2).

LEMma 6.1. A quaternion-Kähler curvature operator with quarter-pinched flag curvature is a multiple of the curvature operator of $\mathbb{H} P^{n}$.

We remark that Berger [B3] proved that a compact quaternion-Kähler manifold with positive sectional curvature must be isometric to the quaternion projective space.

Proof of the theorem. We argue by contradiction. Consider a sequence of orbifolds $M_{k}$ satisfying $\frac{1-\varepsilon_{k}}{4}$-flag pinching, $1 \leq$ Scal $\leq C$ with $\varepsilon_{k} \rightarrow 0$, such that each orbifold violates the conclusion of the theorem. Similarly to Theorem 6.2 , we can find constant $C_{2}$ and $C_{3}$ such that the Ricci flow on $M_{k}$ exists on $\left[0, t_{0}\right]$ with $t_{0}=1 / C_{3}^{2}$ and that $\left(M_{k}, g_{t}\right)$ satisfies the curvature condition $S(t)$, where $S(t)$ is defined as before.

Recall that geodesics are well defined in the orbifold. For any regular point $p_{k} \in M_{k}$ we consider the exponential map exp: $T_{p} M_{k} \rightarrow M_{k}$ and pull back the metric $g_{t}$ of $M_{k}$ to the tangent space. Since we have global curvature bounds, there exists $r>0$ such that this metric is nondegenerate on $B_{r}\left(0_{p_{k}}\right) \subset T_{p_{k}} M_{k}$.

We identify this ball with $B_{r}(0) \subset \mathbb{R}^{n}$ using a linear isometry and denote by $g_{k}\left(p_{k}, t\right)$ the induced Riemannian metric on $B_{r}(0)$. By Shi's estimates we have a priori bounds on all the derivatives of the metric which are independent of $k$ and just depend on a lower bound for $t \in\left(0, t_{0}\right]$. 
We will consider all possible limit metrics $g(t)$ for all convergent subsequences $g_{k}\left(p_{k}, t\right)$ and all choices $p_{k} \in M_{k}$. We can assume that any limit metric comes with the parameter $t$. It is then clear that modulo local diffeomorphism $g(t)$ is a solution to the Ricci flow. All of the limit metrics have nonnegative complex sectional curvature. The strong maximum principle can be utilized to see that the complex sectional curvature is positive unless the limit metric has nongeneric holonomy. Since the sectional curvature is positive the only possible nongeneric holonomies are Kähler, quaternion-Kähler, or Spin(9)-holonomy. It has been known that the Spin(9)-holonomy implies that the limit metric is locally isometric to $\mathbb{C a P}$. Below we subdivide the rest of the proof into three cases, which amounts to $\mathbb{S}^{n}$, $\mathbb{H} P^{n / 4}$ or $\mathbb{C} a P^{2}$, and the weighted complex projective spaces.

Case 1. There is a sequence of points $p_{k} \in M_{k}$ such that a subsequence of $g_{k}\left(p_{k}, t\right)$ converges to a limit metric with generic holonomy.

After passing to a subsequence we may assume $g_{k}\left(p_{k}, t\right)$ itself converges. As explained above the limit metric must have positive complex sectional curvature. This in turn implies that we can find an $\delta>0$ such that the complex sectional of $B_{3 r / 4}\left(p_{k}\right) \subset M_{k}$ is bounded below by $\delta>0$, for large enough $k$. Notice that for all $q_{k} \in B_{5 / 4 r}\left(p_{k}\right)$ the ball of radius $r$ around $q_{k}$ contains a point, on which all complex sectional curvature $>\delta$. This in turn implies that any convergent subsequence $g_{k}\left(q_{k}, t\right)$ has generic holonomy and thus positive complex sectional curvature everywhere. This in turn shows that there exists a $\delta_{2}>0$ such that the complex sectional curvature on the ball $B_{2 r}\left(p_{k}\right)$ is bounded below by $\delta^{\prime}$ for $k$ sufficiently large. Since we have a priori bounds on the diameter of all orbifolds a finite iteration of this argument shows that there exists $k_{0}$ such that the complex sectional curvature of $M_{k}$ is positive for $k \geq k_{0}$, and thus we get a contradiction.

From now on we assume that any limit metric has non generic holonomy.

Claim 1. There are no two sequences $p_{k}, q_{k} \in M_{k}$ such that the metrics $g_{k}\left(p_{k}, t\right)$ and $g_{k}\left(q_{k}, t\right)$ converge to limit metrics with different holonomy groups.

This follows from Case 1. In fact, since the diameters of the orbifolds are bounded above we can choose an fixed integer $l$ and points $p_{k}^{1}, \ldots, p_{k}^{l}$ satisfying $d\left(p_{k}^{i}, p_{k}^{i+1}\right) \leq$ $r / 2, p_{k}^{1}=p_{k}$ and $p_{k}^{l}=q_{k}$.

After passing to subsequence we may assume that $g_{k}\left(p_{k}^{j}, t\right)$ converges to a limit metric $g_{j}$. Suppose now that the holonomies of the limit metrics $g_{j}$ and $g_{j+1}$ are different and both not generic. Suppose for example that $g_{j}$ is Kähler and $g_{j+1}$ is quaternion-Kähler. Then all the curvature operator of $g_{k}\left(p_{k}^{j}, t\right)$ are close to being quaternion-Kähler. Since $B_{r}\left(p_{k}^{j}\right)$ and $B_{k}\left(p_{k}^{j+1}\right)$ have a large intersection a fixed portion of points in $B_{r}(0)$ have curvature operators which are close to ones having quaternion-Kähler with respect to the metric $g_{k}\left(p_{k}^{j}, t\right)$. This in turn shows for certain points in $B_{r}(0)$ the curvature operator (of the limit metric $g_{j}$ ) is both Kähler and quaternion-Kähler. But this is impossible since such a curvature operator would have a vanishing Ricci tensor, a contradiction as the limit metric has positive sectional curvature. 
Hence we below can further assume that all limit metrics have the same holonomy.

Claim 2. The limit metric $g(t)$ on $\left(0, t_{0}\right]$ must be locally isometric to a rank-one symmetric space.

We only need to consider the case that the limit metric has the holomony of a Kähler or quaternion-Kähler manifold since Spin(9)-holonomy implies that the limit metric is locally isometric to $\mathbb{C} a P^{2}$. We only prove it for the case that the limit metric is Kähler, since in view of Lemma 6.1 the argument for quaternionKähler case is analogous (easier). Notice that for $t \rightarrow 0$ the limit metrics have flag pinching constant converging to one quarter which in turn implies that the curvature operator converges to the curvature operator of $\mathbb{C} P^{n / 2}$. The main idea is to establish a maximum principle for the collection of all limit metrics.

We consider for each $t$ the supremum $d(t)$ over the following set: consider for all limit metrics $g(t)$ and all points $x \in B_{r}(0)$ the distance of the curvature operator $\operatorname{Rm}_{g(t)}(x)$ to nonnegative multiples of the curvature operator of $\mathbb{C} P^{n / 2}$ (resp. of $\left.\mathbb{H} P^{n / 4}\right)$. We claim that the supremum of all these distance is actually attained.

In fact to construct a limit metric where the maximum is attained we can argue as follows: Choose for each $k$ a point $q_{k}$ such that the curvature operator $\operatorname{Rm}_{g_{k}(t)}\left(q_{k}\right)$ has maximal distance $d_{k}(t)$ to the $\mathrm{O}(n)$-invariant subset of multiples of the curvature operator of $\mathbb{C} P^{n / 2}$. We choose a regular point $p_{k}$ with $d\left(p_{k}, q_{k}\right)<r / 2$ and pass to subsequence such that $d_{k}(t)$ converges to the supremum and $g_{k}\left(p_{k}, t\right)$ converges to a limit metric $g(t)$. Clearly the above supremum is now attained at some point in the closure of $B_{r / 2}(0) \subset B_{r}(0)$ with this limit metric $g(t)$.

Since the supremum $d(t)$ is attained and $g(t)$ is modulo local diffeomorphisms a solution to the Ricci flow. We can now use the maximum principle to derive that $\lim _{h \rightarrow 0_{+}} \frac{d(t)-d(t-h)}{h} \leq C_{4} d(t)$ holds for some universal constant $C_{4}$. In fact the space of Kähler curvature operators having distance $\leq d(t)$ to multiples of the curvature operators of $\mathbb{C} P^{n}$ form a convex sets. Since the Ricci flow ODE leaves the multiples of $\mathbb{C} P^{n}$ invariant the inequality follows from the dynamical maximum principle and the fact that $\mathrm{Rm}^{2}+\mathrm{Rm}^{\#}$ is locally Lipschitz.

Since $d(t) \rightarrow 0$ as $t \rightarrow 0$ this inequality implies $d(t) \equiv 0$.

Now we can restrict to the cases that all limits are locally isometric to a rank-one symmetric spaces.

Case 2. All limit metrics are locally isometric to $\mathbb{H} P^{n / 4}$ or $\mathbb{C} a P^{2}$ up to scaling.

This implies that all limit metrics have a Bochner-Weitzenböck operator which is positive on two forms. Therefore, the Bochner-Weitzenböck operator of $M_{k}$ on two forms is positive for sufficiently large $k$. As in the odd-dimensional case, it follows that the universal cover $F_{k}$ of the frame bundle of $M_{k}$ has finite second homology. As before we deduce that $F_{k}$ has a priori bound on its injectivity radius and we can assume that $F_{k}$ converges to a limit manifold $F$ endowed with a continuous family of metrics. The quotient $F / \operatorname{Spin}(n)$ is locally isometric to $\mathbb{H} P^{n / 4}$ (or $\mathbb{C} a P^{2}$ ) and since $F$ is simply connected it is globally isometric. Moreover, after passing 
to a subsequence $F_{k}$ endowed with the action of its isometry group is equivariantly diffeomorphic to $F$ endowed with a action of a subgroup of its isometry group.

This shows that $M_{k}$ is diffeomorphic to a quotient of $\mathbb{H} P^{n / 4}$ or $\mathbb{C} a P^{2}$ by a finite isometric group action.

Case 3. All limit metrics are locally isometric to $\mathbb{C} P^{n / 2}$ up to scaling.

We replace $M_{k}$ by a finite cover if needed. It is easy to get a contradiction if the frame bundle $F_{k}$ of $M_{k}$ has finite second homology, since then noncollapsing would imply convergence to the frame bundle of $\mathbb{C} P^{n / 2}$ which has infinite second homology. Thus we assume that there exists a nonzero harmonic two form $\theta$ on $F_{k}$. Since $\mathrm{SO}(n)$ is connected, it induces a trivial action on cohomology and thus $\theta$ is $\mathrm{SO}(n)$-equivariant. Moreover $\theta$ cannot be perpendicular to any pulled back 2 form on $M_{k}$. In fact, for such a form, it is not hard to check that the $L^{2}$-norm of $\nabla \theta$ is bounded below by $\alpha$ times the $L^{2}$ norm of $\theta$, where $\alpha>0$ is independent of $k$. Moreover the number $\alpha$ increases if we scale down the the fibers by a constant. This is a contradiction since the smallest eigenvalue of the Bochner-Weitzenböck operator of $F_{k}$ converges to 0 if we scale down the fibers by small factor $\lambda_{k} \rightarrow 0$. Hence $\theta$ is the pull back of some two form $\eta_{k}$ on $M_{k}$. In particular, we can push down $\theta$ to a class $\eta$ on the orbifold $\left(M_{k}, g_{k}\right)$

For each $\lambda>0$, we scale down the fibers of the frame bundle $F_{k}$ by $\lambda$ and let $\theta_{k, \lambda}$ denote the harmonic two form with respect to this metric representing a fixed cohomology class. The above argument shows that the push down $\eta_{k, \lambda}$ of $\theta_{k, \lambda}$ is of a two form on the orbifold $\left(M_{k}, g_{k}\right)$. Clearly $\eta_{k, \lambda}$ satisfies an elliptic equation and its straightforward to check that $\eta_{k, \lambda}$ converges to a harmonic two form $\omega_{k}$ on $\left(M_{k}, g_{k}\right)$ for $\lambda \rightarrow 0$.

We normalize $\omega_{k}$ to have $L^{2}$-norm equal to $\operatorname{vol}\left(M_{k}\right)$. We may assume that the pull back $c \cdot p r^{*} \omega_{k}$ of $c \omega_{k}$ to $F_{k}$ is a primitive integral class for some $c=c(k)>0$. We consider the $\mathrm{S}^{1}$ bundle over $F_{k}$ whose Euler class is $c \omega_{k}$ and we choose a connection with curvature $c \cdot \omega_{k}$.

Since the curvature when restricted to a $\mathrm{SO}(n)$-fiber vanishes, the $\mathrm{SO}(n)$-action naturally extends to an isometric $\mathrm{SO}(n)$-action of the total space of the $\mathrm{S}^{1}$ bundle. By dividing out the $\mathrm{SO}(n)$-action we obtain an orbifold bundle $\mathrm{S}^{1} \rightarrow S_{k} \rightarrow M_{k}$ over $M_{k}$ with a connection metric whose curvature is given by $c \omega_{k}$. The idea is to show that one can scale the $S^{1}$-fibers such that the curvature pinching constant of the total space approaches 1 as $k \rightarrow \infty$.

This implies that $S_{k}$ endowed with the $\mathrm{S}^{1}$ action is equivariantly diffeomorphic to a space form endowed with a linear $S^{1}$-action and hence the result.

In order to show that the total space has nearly constant curvature for the right choice of scaling of the fibers it suffices to prove $\left\|\nabla \omega_{k}\right\|_{L^{\infty}} \rightarrow 0$. Because this implies that the curvature of the total space $S_{k}$ approaches the curvature of $\mathrm{S}^{1} \rightarrow \mathbb{S}^{2 n+1} \rightarrow \mathbb{C} P^{n}$.

Integrating the harmonic form gives

$$
\int_{M_{k}} \int_{B_{r}\left(0_{p}\right)}\left\|\nabla \omega_{k}\right\|_{\exp (v)}^{2}=\operatorname{vol}\left(B_{r}(0)\right) \int_{M_{k}}\left\|\nabla \omega_{k}\right\|^{2}(p) \leq \operatorname{vol}\left(B_{r}(0)\right) h_{k} \operatorname{vol}\left(M_{k}\right)
$$


where $-h_{k} \rightarrow 0$, as $k \rightarrow \infty$, is the smallest eigenvalue of the Bochner-Weitzenböck operator of $\left(M_{k}, g(t)\right)$ on two forms.

For any $q_{k} \in M_{k}$ we can find a regular point $p_{k}$ with $d\left(p_{k}, q_{k}\right)<r / 2$ and

$$
\int_{B_{r}\left(0_{p_{k}}\right)}\left\|\nabla \omega_{k}\right\|_{\exp (v)}^{2} \leq h_{k} \operatorname{vol}\left(B_{r}(0)\right) \frac{\operatorname{vol}\left(M_{k}\right)}{\operatorname{vol}\left(B_{r / 2}\left(q_{k}\right)\right)} \doteqdot j_{k}
$$

with $j_{k} \rightarrow 0$, where we have used that the $\operatorname{ratio} \operatorname{vol}\left(M_{k}\right) / \operatorname{vol}\left(B_{r / 2}\left(q_{k}\right)\right)$ is bounded.

Since $\exp ^{*} \omega_{k}$ satisfies an elliptic equation with respect to a metric for which we have a priori bounds on all derivatives we deduce that $\exp ^{*} \omega_{k}$ converges to a form which is parallel with respect to the limit metric $g(t)$. This in turn shows that on the orbifold $\left\|\nabla \omega_{k}\right\|_{L^{\infty}} \rightarrow 0$.

Acknowledgments. The first author thanks Nolan Wallach for some helpful discussions. His research is partially supported by a NSF grant DMS-0805834. The paper was written when the second author visited UCSD during February-March of 2009. The second author thanks the mathematical department of UCSD for its hospitality.

\section{References}

[AM] U. Abresch, W.-T. MeYer, A sphere theorem with a pinching constant below 1/4, J. Differential Geom. 44:2 (1996), 214-261.

[AnN] B. Andrews, H. NGuyen, Four-manifolds with 1/4-pinched flag curvature, preprint.

[B1] M. Berger, Sur quelques variétés riemanniennes suffisamment pincées, Bull. Soc. Math. France 88 (1960), 57-71.

[B2] M. Berger, Sur les varites (4/23)-pinces de dimension 5, C. R. Acad. Sci. Paris 257(1963), 4122-4125.

[B3] M. Berger, Trois remarques sur les variétés riemannienes à courbure positive, C. R. Acad. Sci. Paris 263 (1966), 76-78.

[BöW1] C. Böнm, B. Wilking, Nonnegatively curved manifolds with finite fundamental groups admits metrics with positive Ricci curvature, Geom. Funct. Anal. 17 (2007), 665-681.

[BöW2] C. Böhm, B. Wilking, Manifolds with positive curvature operators are space forms, Ann. of Math. (2) 167:3(2008), 1079-1097.

[BrS1] S. Brendle, R. Schoen, Manifolds with 1/4-pinched curvature are space forms, Jour. Amer. Math. Soc. 22:1(2009), 287-307.

[BrS2] S. Brendle, R. Schoen, Classification of manifolds with weakly 1/4-pinched curvatures, Acta Math. 200:1(2008), 1-13.

[C] H. Chen, Pointwise 1/4-pinching 4 manifolds, Ann. Global Geom. 9 (1991), 161176.

[ChL] B. Chow, P. Lu, The maximum principle for systems of parabolic equations subject to an avoidance set, Pacific J. Math. 214:2 (2004), 201-222.

[ChY] B. Chow, D. YANG, Rigidity of nonnegatively curved compact quaternionicKähler manifolds, J. Differential Geom. 29:2 (1989), 361-372.

[FR] F. FAng, X. Rong, Positive pinching, volume and second Betti number, Geom. Funct. Anal. 9:4 (1999), 641-674. 
[G] M. Gromov, Positive curvature, macroscopic dimension, spectral gaps and higher signatures, Functional Analysis on the Eve of the 21st Century, Vol. II (New Brunswick, NJ, 1993), Progr. Math. 132, Birkhäuser Boston, Boston, MA (1996), $1-213$.

[H1] R. Hamilton, Three-manifolds with positive Ricci curvature, J. Differential Geom. 17 (1982), 255-306.

[H2] R. Hamilton, Four-manifolds with positive curvature operator, J. Differenital Geom. 24 (1986), 153-179.

[H3] R. Hamilton, A compactness property for solutions of the Ricci flow, Amer. J. Math. 117:3 (1995), 545-572.

[He] L. Hernández, Kähler manifolds and 1/4-pinching, Duke Math. J. 62:3 (1991), 601-611.

[L] P. LU, A compactness property for solutions of the Ricci flow on orbifolds, Amer. J. Math. 123:6 (2001), 1103-1134.

[MW] M. Micallef, M. WAng, Metrics with nonnegative isotropic curvature, Duke Math. J. 72:3 (1993), 649-672.

[N] H. Nguyen, Invariant curvature cones and the Ricci flow, Int. Math. Res. Notices 2010 (2010), 536-558.

[NiW] L. Ni, J. Wolfson, Positive complex sectional curvature, Ricci flow and the differential sphere theorem, arXiv: 7606.0332 .

[PT] P. Petersen, T. TAO, Classification of almost quarter-pinched manifolds, preprint.

[PeT] A. Petrunin, W. Tuschmann, Diffeomorphism finiteness, positive pinching, and second homology, Geom. Funct. Anal. 9 (1999), 736-774.

[S] J.H. SAmpson, Harmonic maps in Kähler geometry, Harmonic Mappings and Minimal Immersions (Montecatini, 1984), Springer Lecture Notes in Math. 1161 (1985), 193-205.

[Si] Y.-T. SiU, The complex-analyticity of harmonic maps and the strong rigidity of compact Kähler manifolds, Ann. of Math. (2) 112:1 (1980), 73-111.

[W] B. Wilking, Nonnegatively and positively curved manifolds, Surveys in Differential Geometry, Vol. XI, Surv. Differ. Geom., 11, Int. Press, Somerville, MA (2007), $25-62$.

[YZ] S.T. Yau, F. Zheng, Negatively 1/4-pinched Riemannian metric on a compact Kähler manifold, Invent. Math. 103 (1991), 527-535.

LeI Ni, Department of Mathematics, University of California at San Diego, La Jolla, CA 92093, USA

lni@math.ucsd.edu

Burkhard Wilking, Mathematisches Institut, University of Münster, Einsteinstrasse 62, 48149 Münster, Germany

wilking@math.uni-muenster.de

Received: May 28, 2009

Accepted: October 12, 2009

Open Access This article is distributed under the terms of the Creative Commons Attribution Noncommercial License which permits any noncommercial use, distribution, and reproduction in any medium, provided the original author(s) and source are credited. 